\title{
Evaluation and comparison of quality of life and its related factors in pre-dialysis and hemodialysis patients
}

\author{
Mohammad Reza Tamadon ${ }^{1{ }^{\circledR}}$, Sajad Hasani ${ }^{2}$, Ensieh Farhidzadeh ${ }^{3}$, Majid Mirmohammadkhani ${ }^{4}$ \\ ${ }^{1}$ Department of Nephrology, Semnan University of Medical Sciences, Semnan, Iran \\ ${ }^{2}$ Department of Nephrology, Baqiyatallah University of Medical Sciences, Tehran, Iran \\ ${ }^{3}$ Department of Nephrology, Iran University of Medical Sciences, Tehran, Iran \\ ${ }^{4}$ Social Determinants of Health Research Center, Semnan University of Medical Sciences, Semnan, Iran
}

\section{A R T I C L E I N F O}

Article Type:

Original

\section{Article History:}

Received: 7 May 2018

Accepted: 3 July 2018

ePublished: 19 July 2018

\section{Keywords:}

Quality of life

Pre-dialysis

Hemodialysis

Chronic kidney disease

End-stage renal disease

\begin{abstract}
A B S T RACT
Introduction: Chronic kidney disease (CKD) in advanced stages leads to some changes in lifestyle, health status, and personal functioning and consequently affects the patients' quality of life.

Objectives: Given the high prevalence of CKD in the country, this study aimed to determine quality of life and its related factors in this group of patients. Since there has been low focus on quality of life in pre-dialysis stage, this study compared the two groups of pre-dialysis and hemodialysis patients regarding quality of life.

Patients and Methods: This descriptive analytical research was conducted as a cross-sectional study. Using convenience sampling method, we selected a total of 60 kidney failure patients in pre-dialysis stage that referred to nephrology clinics in Semnan and 60 hemodialysis patients who referred to the dialysis center of Kosar hospital in Semnan. Using SF-36 questionnaire and a demographic form, the required data was collected via interviews.

Results: The results showed that the majority of patients had a moderate quality of life. Concerning demographic variables, quality of life had a significant relationship with age, gender, marital status, number of children, employment status, education level, income level, hemoglobin, underlying disease, duration of disease, and duration of dialysis. Moreover, patients in pre-dialysis stage had a better score than hemodialysis patients regarding the overall quality of life $(P \leq 0.05)$.

Conclusion: This study showed, a design a framework for care services provided by medical staffs and supports provided by insurance organizations and other institutions is necessary. The framework must help to improve quality of life of patients and prevent the deterioration of quality of life in advanced stages of the disease.
\end{abstract}

\section{Implication for health policy/practice/research/medical education:}

CKD has high prevalence and quality of life and its related factors are important issues in this group of patients. Since there has been low focus on quality of life in pre-dialysis stage, this study compared the two groups of pre-dialysis and hemodialysis patients in regarding quality of life. It is necessary to design a framework for care services provided by medical staffs and supports provided by insurance organizations and other institutions.

Please cite this paper as: Tamadon MR, Hasani S, Farhidzadeh E, Mirmohammadkhani M. Evaluation and comparison of quality of life and its related factors in pre-dialysis and hemodialysis patients. J Nephropharmacol. 2018;7(2):114-121.

\section{Introduction}

Chronic kidney disease (CKD) is a threat to public health throughout the world and its prevalence is increasing, especially in adults aged 60 years and older $(1,2)$. It is estimated that more than $10 \%$ of adult population in the United States, that includes twenty million people of the total population of the US, are affected by CKD (3). In Iran there is no exact statistical data on the number of
CKD patients. However, based on a national report in 2004, the incidence of CKD among the total population of the country is 700000 and every year more than $2 \%$ of Iran's population is added as well $(4,5)$. Patients with CKD are affected by varying degrees of the disease (zero to five) that are measured based on the level of glomerular filtration rate (GFR) declinE (6). The clinical and laboratory signs are highlighted in stage 3 and 4 
patients. In addition, stage 5 patients usually have obvious complications in daily activities, health, nutritional status, and homeostasis of water and electrolytes. Such patients finally develop uremic syndrome that leads to death if remains untreated (7). Dialysis and kidney transplant are renal replacement therapies that are applied for patients with end-stage renal disease. These methods can improve the patient's health status and protect their lives. However in advanced stages of the disease the patients are faced with many physical, psychological, and social problems $(1,8-10)$. Clinical complications such as muscle cramps, nausea, vomiting, dizziness, anorexia, anemia, shortness of breath, sleep disturbances, fatigue, as well as dependence to others, decreased self-esteem, and loneliness are among the physical and psychological complications which reduce the patients' quality of life $(8,11)$. Hence, today the medical care services are aimed not only to increase the life time of CKD patients but also to improve quality of life of the patients. Thus, the promotion of quality of life has become one of the main goals of the medical community and has attracted their attention (9).

According to the World Health Organization (WHO), quality of life is defined as an individual's perception of their position in life in the context of the culture and value system in which they live and in relation to their goals, expectations and standards and concerns (12). According to the views of experts, quality of life is a feeling of wellbeing that is rooted in satisfaction or dissatisfaction with various important aspects of an individual's life. A sense of security, personal beliefs, emotional conflicts, objectives, and level of failures tolerance altogether determine an individual's perceptions of oneself. In fact, when someone feels happy and satisfied with its own life and does not feel discomfort at the time of illness, he/she will have more energy to take care of its own health. When a person is good in self-care, he/she will live healthier and therefore will have a better quality of life $(13,14)$.

Studies have shown that poor quality of life is a factor effective in the development of cardiovascular diseases, recurrent hospitalizations, and mortality of the patients )15(. Evaluation of quality of life in these patients is an important criterion for determining the effectiveness of health care services that can help to plan medical and supportive care services and would be utilized as an appropriate approach to reduce the adverse effects of the disease (16). Therefore, evaluating the quality of life of patients with CKD and determining its different stages is of great importance. Several studies have investigated and determined the quality of life and its related factors in CKD patients and their results suggested low levels of quality of life, as compared with the healthy subjects $(10,17,18)$. According to the results of these studies, among the important factors associated with quality of life we may note age, gender, occupation, marital status, income level, concurrent disease, education level, hemoglobin level, duration of dialysis therapy, and stage of $\operatorname{CKD}(8,10,19,20)$. However, there are conflicting results about the relationship between these factors and the levels of quality of life. For instance, some of the studies have reported that factors such as gender, being single, and low level of education are among the factors adversely affecting quality of life. However, such a finding is not consistent with the results of similar studies in other regions (21-23).

\section{Objectives}

So far, previously conducted studies have paid little attention to quality of life of patients in pre-dialysis stage. In addition, the relationship between the potential factors and quality of life of patients with CKD might have been affected by different geographical and cultural factors and types of instruments used. The present study aimed to investigate quality of life of patients with CKD in Semnan through using SF-36 questionnaire at different stages of disease (pre-dialysis, hemodialysis stage). It was also aimed to identify potential risk factors affecting quality of life of these patients to provide an appropriate ground for improving quality of life of this group of patients.

\section{Patients and Methods}

\section{Study population and research design}

This descriptive analytical study was conducted on predialysis patients with CKD who referred to nephrology clinics and hemodialysis patients who referred to dialysis center of Kosar hospital in Semnan from September 2015, to March 2016. Convenience sampling method was used to select the patients in pre-dialysis stage. In addition, in order to randomly select the hemodialysis patients, we used the list of all dialysis patients who referred to Kosar hospital. The selected patients met the laboratory and clinical requirements and conditions to be allocated to one of the two groups of pre-dialysis and hemodialysis. Moreover, patients with a history of mental illness, cancer, and other severe and limiting diseases were excluded.

The eligible patients who met the inclusion criteria were informed about the goals of the study. After obtaining informed consent, the questionnaire was completed by an interview at the time of patient's admission to the clinic or dialysis center. To enroll pre-dialysis patients into the study, the list of these patients and their files were reviewed by the researcher and their records at the clinic were extracted. Some of the patients had an appointment to visit the clinic during the period of study. At the time of their visits to the clinic, the questionnaire was completed via face to face interview. However, some other patients have no appointments. They were called by phone and were invited to visit the researcher where patients desired or were more convenient for them (clinic or hospital). To collect the required data from dialysis patients, the researcher visited the dialysis center and obtained the necessary data. The questionnaire was completed for all the patients via interviews. 
A researcher-made questionnaire was used to collect demographic data as well as medical profile. The questionnaire included items to collect data on demographic characteristics including gender, age, Blood type, underlying diseases, marital status, number of children, residence status, employment status, education level, duration of disease (for pre-dialysis patients), duration of dialysis (for dialysis patients), hemoglobin level, and income level. To evaluate quality of life of the studied patients we used SF36 questionnaire. This questionnaire includes 36 questions to assess the quality of life in eight domains including the followings; general health (6 items), physical functioning (10 items), role limitations due to physical health ( 4 items), role limitations due to emotional problems ( 2 items), social functioning (2 items), vital force and energy (4), bodily pain (2 items), and mental health of people (5 questions). All questions have a Likert type scale ranging from zero to five (where zero indicates the worst and five indicates the best possible state for every individual). Total scores of questions range from zero to 100. Based on the responses of the patients, their quality of life was classified as "good" (between the 75th percentile and higher), "somewhat favorable" or "moderate" (between 25th and 75th percentile), and "bad" (less than 25th percentile). The validity and reliability of this questionnaire had already been proved by various studies. In the present study, the validity of the tool was proved based on the results of other similar studies and comments by the experts. In addition, in order to test the reliability of the tool, it was run on 20 students and the Cronbach's alpha was measured $(\alpha=0.83)$.

\section{Ethical issues}

The research followed the tenets of the Declaration of Helsinki. Informed consents were obtained from all patients. The study was approved by the Social Determinants of Health Research Center, Semnan University of Medical Sciences (\#A-10-23-2). This paper is extracted from general practitioner thesis of Sajad Hasani, in the Department of Internal Medicine, Semnan University of Medical Sciences (Ref. No: 496).

\section{Statistical analysis}

To analyze the data, first, the collected data were entered into SPSS version 20 software. Descriptive statistics were used to describe the data. On the other hand, the relationship between the variables was studied using analytical statistics including Kolmogorov-Smirnov test (to determine normality), parametric tests including $t$ test, analysis of variance (ANOVA), non-parametric test including Mann-Whitney test and Kruskal Wallis test. The significance level was set at $5 \%$.

\section{Results}

A total of 120 patients, including 60 patients at predialysis stage and 60 dialysis patients were studied. Of all the patients in both groups, 62 persons $(51.7 \%)$ were male and the rest were female $(n=58,48.3 \%)$. Among all the age group, the highest frequency of patients $(n=44$, $36.7 \%$ ) was observed in the age group 60 years and the lowest frequency of patients $(n=5,4.2 \%)$ was observed in the age group 29 years and younger. Of all, 91 patients (75.8\%) were married. The most frequent number of children was "three children and less" which was reported by 68 patients $(56.7 \%)$. Of all the patients, 102 patients (85\%) were employed. Primary education was the most frequent education level $(n=39,32.5 \%)$ among all the patients. The majority of patients $(\mathrm{n}=93,77.5 \%)$ had poor or very poor income level, while 96 persons (80\%) were living in urban areas and the rest $(n=24,20 \%)$ were living in rural areas. Hemoglobin level lower than 11 was the most prevalent level of hemoglobin among the studied patients $(n=64,53.3 \%)$. The maximum duration of the disease was more than 60 months $(n=50,41.7 \%)$. Hypertension $(n=78,65 \%)$ and diabetes $(n=62,51.7 \%)$, respectively, were the most prevalent underlying diseases reported by the studied patients. The blood types $\mathrm{O}(\mathrm{n}$ $=43,36.1 \%)$ and blood type $\mathrm{B}(\mathrm{n}=38,31.9 \%)$ were the most prevalent Blood types. Among dialysis patients, 40 patients $(66.6 \%)$ were on dialysis for three years or less, while 14 patients $(23.4 \%)$ were on dialysis for 4 to 9 years, and only 6 patients (10\%) were on dialysis for 10 years and more. According to the results of our evaluation, the demographic and medical characteristics including age, gender, marital status, number of children, employment status, education level, income, place of residence, hemoglobin level, duration of illness, and Blood types were not significantly different $(P>0.05)$. Tables 1 and 2 present the distribution of demographic and medical characteristics of the studied patients by pre-dialysis and hemodialysis groups.

Mean (SD) total score of quality of life of all patients was $68.78 \pm 13.39$. Among all the dimensions of quality of life, the highest score was related to emotional role functioning (100 points). The two domains of general health $(58 \pm 17.15)$ in pre-dialysis patients and physical functioning $(47.25 \pm 24.41)$ in dialysis patients had the lowest scores. Pre-dialysis patients, as compared with dialysis patients, had significantly higher mean (SD) scores in the domains of total quality of life, physical functioning, social functioning, energy/fatigue, bodily pain, and general health $(P<0.05)$. However, there was no significant difference between the two groups regarding physical role functioning, emotional role functioning, and mental health $(P>0.05)$. Table 3 presents mean and standard deviation of scores obtained for total quality of life and its different domains in the two groups of predialysis and dialysis patients.

We evaluated the relationship between total quality of life and the factors affecting it. The results of evaluation in pre-dialysis patients showed that several factors including age, marital status, employment status, education level, 
Table 1. Distribution of demographic characteristics of patients in the two groups of pre-dialysis and hemodialysis

\begin{tabular}{|c|c|c|c|c|}
\hline Variables & & Pre-dialysis patients, $n=60$ & Hemodialysis patients, $n=60$ & $P$ value \\
\hline \multirow{5}{*}{ Age } & $\geq 29$ & 0 & $5(8.3 \%)$ & \multirow{5}{*}{0.521} \\
\hline & $30-39$ & $9(15 \%)$ & $8(13.3 \%)$ & \\
\hline & $40-49$ & $14(23.3 \%)$ & $12(20 \%)$ & \\
\hline & $50-59$ & $15(25 \%)$ & $13(21.7 \%)$ & \\
\hline & $\geq 60$ & $22(36.7 \%)$ & $22(36.7 \%)$ & \\
\hline \multicolumn{2}{|l|}{ Being male } & $28(46.7 \%)$ & $34(56.7 \%)$ & \multirow{6}{*}{0.652} \\
\hline \multirow{3}{*}{ Marital status } & Single & $3(5 \%)$ & $8(13.3 \%)$ & \\
\hline & Married & $49(81.7 \%)$ & $42(70 \%)$ & \\
\hline & Widowed & $8(13.3 \%)$ & $10(16.7 \%)$ & \\
\hline \multirow{2}{*}{ Number of children } & $3 \geq$ & $43(6 / 71 \%)$ & $40(7 / 66 \%)$ & \\
\hline & $3<$ & $17(4 / 28 \%)$ & $20(8 / 28 \%)$ & \\
\hline \multicolumn{2}{|l|}{ Employment } & $11(18.3 \%)$ & $7(11.7 \%)$ & 0.711 \\
\hline \multirow{4}{*}{ Education level } & Illiterate & $9(15 \%)$ & $8(13.3 \%)$ & \multirow{4}{*}{0.541} \\
\hline & Primary school & $18(30 \%)$ & $21(35 \%)$ & \\
\hline & High school diploma & $26(4 / 43 \%)$ & $27(45 \%)$ & \\
\hline & Academic & $7(11.7 \%)$ & $4(6.7 \%)$ & \\
\hline \multirow{4}{*}{$\begin{array}{l}\text { Income rate } \\
\text { (Toman) }\end{array}$} & $<500$ thousand (very poor) & 0 & $2(3.3 \%)$ & \multirow{4}{*}{0.241} \\
\hline & 500 thousand -1 million (poor) & $44(73.3 \%)$ & $47(78.3 \%)$ & \\
\hline & 1 million - 1.5 million (moderate) & $16(26.7 \%)$ & $11(18.3 \%)$ & \\
\hline & > 1.5 million (good) & 0 & 0 & \\
\hline \multicolumn{2}{|l|}{ Living in urban areas } & $43(71.7 \%)$ & $53(88.3 \%)$ & 0.340 \\
\hline
\end{tabular}

Table 2. Distribution of medical characteristics in the two groups of pre-dialysis and dialysis patients

\begin{tabular}{lccc}
\hline Variables & $\begin{array}{c}\text { Pre-dialysis } \\
\text { patients, n=60 }\end{array}$ & $\begin{array}{c}\text { Hemodialysis } \\
\text { patients, } \mathbf{n = 6 0}\end{array}$ & P value \\
\hline Hemoglobin level & & & \\
$<11$ & $26(43.3 \%)$ & $38(63.3 \%)$ & 0.130 \\
$\geq 11$ & $34(56.3 \%)$ & $22(7 / 36 \%)$ & \\
Duration of disease & & & \\
$<12$ months & $13(21.7 \%)$ & $9(15 \%)$ & \\
$13-48$ months & $24(40 \%)$ & $19(31.7 \%)$ & 0.214 \\
$49-60$ months & $1(1.7 \%)$ & $4(6.7 \%)$ & \\
$>60$ months & $22(36.7 \%)$ & $28(46.7 \%)$ & \\
Underlying disease & & & \\
Hypertension & $40(66.7 \%)$ & $38(63.3 \%)$ & \\
Diabetes & $35(58.3 \%)$ & $27(45 \%)$ & \\
Other & $6(10 \%)$ & $8(13.3 \%)$ & \\
Blood group & & & \\
A & $6(10.2 \%)$ & $13(21.7 \%)$ & \\
B & $25(42.4 \%)$ & $13(21.7 \%)$ & 0.101 \\
AB & $11(18.6 \%)$ & $8(13.3 \%)$ & \\
O & $17(28.8 \%)$ & $26(43.3 \%)$ & \\
\hline
\end{tabular}

income level, duration of disease, underlying diseases, and hemoglobin level were significantly associated with better quality of life. Accordingly, patients aged between 30-39 years old, employed, single, with academic education, with a moderate average income, with disease duration less than 12 months, without diabetes, and with hemoglobin level of 11 and higher had obtained better quality of life scores. Nevertheless, the total score of quality of life in dialysis patients was only associated with employment status, income level, and duration of dialysis. Accordingly, patients with a job and moderate income level who had been under dialysis treatment for three years and less had better quality of life scores.

Moreover, we evaluated the relationship between different domains of quality of life and the factors affecting them. The results of our evaluation in pre-dialysis patients showed a significant relationship between gender and two domains of mental health and social functioning. Accordingly, women obtained significantly higher scores, as compared with men. There was a significant relationship between physical functioning and being 29 years or younger. In addition, married people were in better condition in terms of energy - fatigue domain. Patients with three children or less obtained better scores for the two domains of bodily pain and physical functioning while people with three children or more obtained better scores for the domain of mental health. Employed patients had better scores in the domains of energy - fatigue, mental health, social functioning, and general health. Moreover, in domains of physical functioning, energy - fatigue, mental health, and general health people with moderate income level received higher scores than those with low income levels. Patients without diabetes had higher scores in domains of physical functioning, mental health, bodily pain, and general health. People with hemoglobin level of 11 and higher had better scores in domains of physical functioning and general health. No significant relationship was observed between other domains of quality of life and other factors.

Among the dialysis patients, the patients who were married or widowed had higher scores in the domain of physical role functioning (100 points), as compared with the single patients. The dialysis patients aged between 
Table 3. Mean and standard deviation of scores obtained for total quality of life and its different domains in the two groups of pre-dialysis and dialysis patients

\begin{tabular}{|c|c|c|c|}
\hline Quality of life and its domains & $\begin{array}{l}\text { Pre-dialysis patients, } n=60 \\
\text { Mean } \pm \text { SD }\end{array}$ & $\begin{array}{l}\text { Hemodialysis patients, } n=60 \\
\text { Mean } \pm \text { SD }\end{array}$ & $P$ value \\
\hline Total quality of life & $75.59 \pm 9.38$ & $61.98 \pm 13.40$ & $<0.001$ \\
\hline Physical functioning & $68.75 \pm 22.08$ & $47.25 \pm 24.41$ & $<0.001$ \\
\hline Physical role functioning & $100 \pm 0.0$ & $99.58 \pm 3.22$ & 0.319 \\
\hline Emotional role functioning & $100 \pm 0.0$ & $100 \pm 0.0$ & - \\
\hline Fatigue/Energy & $68.58 \pm 12.79$ & $58.25 \pm 18.01$ & $<0.001$ \\
\hline Mental health & $70 \pm 16.45$ & $64.60 \pm 19.93$ & 0.108 \\
\hline Social role functioning & $86.45 \pm 13.07$ & $70.20 \pm 25.53$ & $<0.001$ \\
\hline Bodily pain & $87.20 \pm 12.58$ & $55.85 \pm 31.33$ & $<0.001$ \\
\hline General health & $58 \pm 17.15$ & $40.08 \pm 22.27$ & $<0.001$ \\
\hline
\end{tabular}

30 to 39 years old had higher scores in the two domains of physical functioning and physical role functioning. The employed dialysis patients obtained better scores in domains of physical functioning, energy - fatigue, and general health. Dialysis patients without hypertension, as compared with those with hypertension, had higher scores in two domains of bodily pain and general health. Patients with disease duration of 1 year or less had better general health scores. In addition, patients with disease duration of 1 year or less obtained higher scores in domains of physical functioning and general health. No significant relationship was observed between other domains of quality of life and other factors.

\section{Discussion}

This study aimed to determine the level of quality of life in patients with CKD and compare the two groups of predialysis and hemodialysis patients in terms of the scores of quality of life and related factors. The results of this study can be utilized to provide proper education and support for nurses and other health care workers and families who provide care services for patients. Such efforts can also improve quality of life of this group of patients.

The results of analytical analysis showed that patients in pre-dialysis stage obtained better scores for total quality of life, as compared with hemodialysis patients. This finding is consistent with the results of a study by Mujais et al, which showed that quality of life of patients with CKD is associated with the stage of the disease. Accordingly, in more advanced stages of CKD, there was a more significant reduction in quality of life (24). Although end stage CKD patients on hemodialysis are saved from certain death, apparently during the disease progress they are affected by a wide range of physical, psychological, social, and economic problems which in turn will affect their quality of life (21). Based on the results of this study, with advances in stages of disease, quality of life of dialysis patients was low in the domains of physical functioning, social functioning, energy/fatigue, bodily pain, and general health. It seems that the initiation of dialysis affected these domains more than the other ones. Considering this finding, it is necessary to pay special attention to physical functioning of dialysis patients. In order to utilize an appropriate approach for reducing adverse complications, it is recommended to improve the condition of patients during dialysis and identify new ways to enhance quality of life of this group of patients. In addition, it might be also helpful to protect pre-dialysis patients against more advanced stages of disease through utilizing newer and more accurate treatment methods.

The results of this study showed that in the two domains of mental health and social functioning the scores obtained by women in pre-dialysis group were better than the scores obtained by men. However, in dialysis patients there was no significant relationship between gender and the mentioned domains. In a study by Al Wakeel et al which was conducted on quality of life in patients treated with peritoneal dialysis and hemodialysis, men had lower quality of life (25). In a study by Bayoumi et al, the researchers investigated quality of life of 100 hemodialysis patients and the same result was obtained (26). On the contrary, the study by Edalat-Nejad et al and the study of Rafiei et al showed no relationship between gender and quality of life in hemodialysis patients $(27,28)$. Given the conflicting results of studies which investigated the relationship between gender and quality of life of CKD patients, and based on the results of this study, apparently in the more advanced stages of the disease a more important factors other than gender, such as the initiation of dialysis, which is effective in determining quality of life was existed.

According to the results of the present study, in predialysis patients younger age was associated with higher scores of total quality of life and physical functioning. However, in dialysis patients older age was associated with lower scores of physical functioning domain. In three studies conducted in Saudi Arabia, China, and the United States, older age was among the negative factors affecting quality of life in dialysis patients $(24,25,29)$. However, in a study in Tehran which investigated the quality of life of 202 CKD patients, there was no significant relationship between age and quality of life (28). Due to inconsistencies 
in the results of previous studies, probably quality of life of patients with chronic disease in later stages of life is not only associated with age, but also is affected by other factors such as diseases and concurrent disabilities, social limits, psychological stress, loneliness, and lack of support. Thus, it is recommended to conduct further similar studies to determine the underlying factors affecting quality of life of older people.

Various studies have investigated the relationship between quality of life and marital status, but their results are conflicting. Some of these studies indicate that being married is associated with better quality of life while others expressed opposite results $(19,21,22)$. The results of this study showed that lower number of children in both groups of patients was associated with better scores in domains of physical functioning and bodily pain. In pre-dialysis group, patients with more than three children had better mental health scores. It seems that families and children act as sources of support for patients with chronic disease and thus reduce depression and improve mental health (28). However having a child was not necessarily effective in improving quality of life of patients in the physical domains. Based on the results of this study, the higher education level in pre-dialysis patients was associated with more favorable quality of life, especially in the physical domains. This relationship is also confirmed in a study by Wu et al (29).

In this study, employment played an important role in improving quality of life of CKD patients and similar studies could confirm this finding. In a study by Porter et al, unemployment was associated with quality of life of patients with CKD (30). In a study by Bayoumi et al unemployment was one of the factors adversely affecting quality of life (26). However, in a study by Rafiei et al in Iran, no significant relationship between employment status and quality of life was detected (28). However, apparently with the initiation of dialysis in end-stage CKD patients, they are faced with disability in work or job loss, which can deteriorate the economic condition of this group of patients and make a direct impact on their quality of life. Since the results of this study and many other similar studies indicate a relationship between employment and quality of life, the development of support programs to improve the employment status of patients can be helpful.

Consistent with the study by Rafiei et al (28), in our study, moderate level of income was associated with better quality of life in both groups of patients. Patients who had low or very low income had lower total scores in different domains of quality of life. The medical services used for the treatment of advanced stages of CKD, such as hemodialysis and kidney transplantation and frequent hospitalizations, are expensive. Thus, insurance organizations and other institutions must provide more financial supports to improve the quality of life of this group of patients.

Concerning underlying diseases, diabetes was more effective than hypertension and other underlying diseases in reducing the quality of life of pre-dialysis patients. Previous studies have reported conflicting results on the relationship between diabetes and quality of life $(24,31)$. In addition, no significant relationship between diabetes and quality of life in hemodialysis patients was seen. On the contrary, in this group of patients, hypertensive was significantly associated with reduced quality of life in the domains of public health and bodily pain. This finding is consistent with the results of a study by Porter et al (30). The results of this study showed that higher levels of serum hemoglobin in CKD patients was associated with better quality of life, especially in domains of physical functioning and general health. This association was significant in pre-dialysis patients. This finding is consistent with the results of a study by Perlman et al (32). In addition, the results of the study by Mujais et al, showed that anemia and low hemoglobin were among the major factors affecting quality of life in CKD patients in the final stages of the disease (24). Considering the results of this study and other similar studies, therapeutic interventions to increase and maintain hemoglobin level in patients with CKD can be useful in improving the quality of life of this group of patients. Thus, it is recommended to conduct interventional studies in this area.

In CKD patients, duration of disease was inversely related to quality of life; this relationship was observed in the domains of physical functioning, bodily pain, and general health. Moreover, the duration of dialysis in patients undergoing hemodialysis was associated with their quality of life. In other words, it had an inverse relationship with the domains of physical functioning and general health. These results are consistent with the findings of some other similar studies $(25,26,31)$. However, in other studies that have been conducted in Iran, no significant relationship between duration of dialysis and quality of life is reported; thus their results are not consistent with our finding $(27,28)$. Based on the results of the study, it seems that with the passage of time people become more adapted to their disease, however because of disease and treatment complications which gradually emerge, people will suffer from higher levels of disability and face more social limits, economic tensions, and mental stresses. Such conditions can lower patients' satisfaction with life and decrease their functioning and quality of life. Therefore, several measures, such as culture building for organ donation and the provision of suitable alternative treatments such as kidney transplant, can be utilized to increase the duration of the disease and help to improve the quality of life for this group of patients.

According to the results of the present study, the majority of patients $(80 \%)$ were living in urban areas and there was no significant relationship between place of residence and quality of life. Although people living in rural areas have more limited access to health centers, hospitals, and amenities, they are not necessarily the determinants of 
quality of life. In urban areas, there are some problems such as air pollution and low physical activity which can affect a person's quality of life. Therefore, it is recommended to conduct further similar studies on this subject.

The results of the study showed no significant relationship between blood type and quality of life. In our study, blood type $\mathrm{O}$ was the most common blood type in the studied patients. On the other hand, other studies did not pay much attention to this issue. Thus, it is recommended to conduct further similar studies on the relationship between blood type and quality of life. In addition, given the results of this study and other similar which indicated the effects of known factors on quality of life of CKD patients, it is suggested to conduct interventional studies to eliminate some of the factors that negatively affect the quality of life.

\section{Conclusion}

Overall, the findings of this study showed that in the majority of patients with CKD the mean score of quality of life was at a good level (between 25th to 75th percentiles). Moreover, pre-dialysis patient, as compared with patients who were on hemodialysis, had a higher quality of life. This study showed that different factors including age, marital status, employment status, education level, income level, duration of disease, underlying disease, and hemoglobin level could affect the quality of life of patients with CKD.

\section{Limitations of the study}

This study was conducted on a limited sample size and needs further confirmation by larger studies.

\section{Authors' contribution}

MRT supervised the research. EF and SH conducted the investigation. MM prepared the primary draft. MRT finalized the final manuscript. All authors read and signed the final paper.

\section{Conflicts of interest}

There were no points of conflicts to declare.

\section{Ethical considerations}

Ethical issues (including plagiarism, data fabrication, double publication) have been completely observed by the authors.

\section{Funding/Support}

This paper is extracted from the general practitioner thesis and Semnan University of Medical Sciences provided financial provided support for this research.

\section{References}

1. Okubo R, Kai H, Kondo M, Saito C, Yoh K, Morito N, et al. Health-related quality of life and prognosis in patients with chronic kidney disease: a 3-year follow-up study. Clin Exp Nephrol. 2014;18:697-703. doi: 10.1007/s10157-013- 0901-x.

2. TonelliM, Riella M. Chronic kidney disease and the ageing population. J Ren Care. 2014;40:1-5.

3. CDC. National chronic kidney disease fact sheet. Atlanta: Available from: https://www.cdc.gov/kidneydisease/pdf/ kidney_factsheet.pdf. 2014.

4. Nafar M, Mousavi SM, Mahdavi M, Pour-Reza-Gholi F, Firoozan A, Einollahi B, et al. Burden of Chronic Kidney Disease in Iran. Iran J Kidney Dis. 2008;2:183-92.

5. Tohidi M, Hasheminia M, Mohebi R, Khalili D, Hosseinpanah F, Yazdani B, et al. Incidence of chronic kidney disease and its risk factors, results of over 10 year follow up in an Iranian cohort. Plos One. 2012;7:e45304. doi: 10.1371/journal.pone.0045304

6. Wyld ML, Lee CM, Zhuo X, White S, Shaw JE, Morton RL, et al. Cost to government and society of chronic kidney disease stage 1-5: a national cohort study. Intern Med J. 2015;45:741-7. doi: 10.1111/imj.12797.

7. Hajivandi A, Amiri M. World kidney day 2014: Kidney disease and elderly. J Parathyr Dis. 2014; 2:3-4.

8. Davison SN, Jhangri GS. The relationship between spirituality, psychosocial adjustment to illness, and healthrelated quality of life in patients with advanced chronic kidney disease. J Pain Symptom Manage. 2013;45:170-8. doi: 10.1016/j.jpainsymman.2012.02.019.

9. Poppe C, Crombez G, Hanoulle I, Vogelaers D, Petrovic M. Improving quality of life in patients with chronic kidney disease: influence of acceptance and personality. Nephrol Dial Transplant. 2013;28:116-21. doi: 10.1093/ndt/gfs151.

10. Tong A, Wong G, McTaggart S, Henning P, Mackie F, Carroll RP, et al. Quality of life of young adults and adolescents with chronic kidney disease. J Pediatr. 2013;163:1179-85.e5. doi: 10.1016/j.jpeds.2013.04.066.

11. Al-Arabi S. Quality of life: subjective descriptions of challenges to patients with end stage renal disease. Nephrol Nurs J. 2006;33:285-92.

12. Fayers PM, Machin D. Quality of life: the assessment, analysis and interpretation of patient-reported outcomes: John Wiley \& Sons; 2013.

13. Sammarco A. Perceived social support, uncertainty, and quality of life of younger breast cancer survivors. Cancer Nurs. 2001;24:212-9.

14. Ragonesi PD, Ragonesi G, Merati L, Taddei M. The impact of diabetes mellitus on quality of life in elderly patients. Arch Gerontol Geriatr. 1998;26:417-22.

15. dos Reis Santos I, Danaga AR, de CarvalhoAguiar I, Oliveira EF, Dias IS, Urbano JJ, et al. Cardiovascular risk and mortality in end-stage renal disease patients undergoing dialysis: sleep study, pulmonary function, respiratory mechanics, upper airway collapsibility, autonomic nervous activity, depression, anxiety, stress and quality of life: a prospective, double blind, randomized controlled clinical trial. BMC Nephrol. 2013;14:215. doi: 10.1186/1471-236914-215.

16. Fukuhara S, Lopes AA, Bragg-Gresham JL, Kurokawa K, Mapes DL, Akizawa T, et al. Health-related quality of life among dialysis patients on three continents: the Dialysis Outcomes and PracticePatterns Study. Kidney Int. 2003;64:1903-10.

17. Wyld M, Morton RL, Hayen A, Howard K, Webster AC. A systematic review and meta-analysis of utility-based quality of life in chronic kidney disease treatments. PLoS Med. 
2012:9:e1001307.

18. Pagels AA, Söderkvist BK, Medin C, Hylander B, Heiwe S. Health-related quality of life in different stages of chronic kidney disease and at initiation of dialysis treatment. Health Qual Life Outcomes. 2012;10:71. doi: 10.1186/1477-752510-71.

19. Vosughi M, Movahedpour A. Comparison quality of life between hemodialysis and transplant patient in Ardabil medical centers. Ardabil Univ Med Sci J. 2009;9:171-9.

20. Kimmel PL, Patel SS. Quality of life in patients with chronic kidney disease: focus on end-stage renal disease treated with hemodialysis. Semin Nephrol. 2006;26:68-79.

21. Tayyebi A, Salimi SH, Mahmoudi H, Tadrisi SD. Comparison of quality of life in haemodialysis and renal transplantation pateints. J Crit Care Nurs. 2010;3:19-22.

22. Fujisawa M, Ichikawa $\mathrm{Y}$, Yoshiya K, Isotani S, Higuchi A, Nagano S, et al. Assessment of health-related quality of life in renal transplant and hemodialysis patients using the SF36 health survey. Urology. 2000;56:201-6.

23. Unruh ML, Hess R. Assessment of health-related quality of life among patients with chronic kidney disease. Adv Chronic Kidney Dis. 2007;14:345-52.

24. Mujais SK, Story K, Brouillette J, Takano T, Soroka S, Franek $\mathrm{C}$, et al. Health-related quality of life in CKD patients: correlates and evolution over time. Clin J Am Soc Nephrol. 2009;4:1293-301.

25. Al Wakeel J, Al Harbi A, Bayoumi M, Al-Suwaida K, Al Ghonaim M, Mishkirye A. Quality of life in hemodialysis and peritoneal dialysis patients in Saudi Arabia. Ann Saudi Med. 2012;32:570-4. doi: 10.5144/0256-4947.2012.570.

26. Bayoumi M, Al Harbi A, Al Suwaida A, Al Ghonaim M, Al Wakeel J, Mishkiry A. Predictors of quality of life in hemodialysis patients. Saudi J Kidney Dis Transpl. 2013;24:254-9.

27. Edalat-Nejad M, Qlich-Khani M. Quality of life and sleep in hemodialysis patients. Saudi J Kidney Dis Transpl. 2013;24:514-8

28. Rafii F, Rambod M, Hosseini AF. Quality of life in end stage renal disease and its related factors. Iran J Nurs. 2010;23:3542.

29. Wu F, Cui L, Gao X, Zhou H, Yang M, Pan J, et al. Quality of life in peritoneal and hemodialysis patients in China. Ren Fail. 2013;35:456-9. doi: 10.3109/0886022X.2013.766573.

30. Porter A, Fischer MJ, Brooks D, Bruce M, Charleston J, Cleveland WH, et al. Quality of life and psychosocial factors in African Americans with hypertensive chronic kidney disease. Transl Res. 2012;159:4-11. doi: 10.1016/j. trsl.2011.09.004.

31. Anees M, Hameed F, Mumtaz A, Ibrahim M, Khan MNS. Dialysis-related factors affecting quality of life in patients on hemodialysis. Iran J Kidney Dis. 2011;5:9-14.

32. Perlman RL, Finkelstein FO, Liu L, Roys E, Kiser M, Eisele $\mathrm{G}$, et al. Quality of life in chronic kidney disease (CKD): a cross-sectional analysis in the Renal Research InstituteCKD study. Am J Kidney Dis. 2005;45:658-66.

Copyright $\odot 2018$ The Author(s); Published by Society of Diabetic Nephropathy Prevention. This is an open-access article distributed under the terms of the Creative Commons Attribution License (http://creativecommons.org/licenses/by/4.0), which permits unrestricted use, distribution, and reproduction in any medium, provided the original work is properly cited. 